International Journal of Soft Computing 7 (4): 217-223, 2012

ISSN: 1816-9503

C) Medwell Journals, 2012

\title{
Simulation of Improved Hybrid Petri Nets Intersection Model Considering Traffic Distribution
}

\author{
M.S. Harjono, A. Halim and K. Ramli \\ Department of Electrical Engineering, Faculty of Engineering, \\ Universitas Indonesia, 16424 Depok, Indonesia
}

\begin{abstract}
Traffic Control System in an intersection is a basis for management of urban traffic area system in particular traffic safety gain more attention now a days. This study proposed a new hybrid petri nets structure at the intersection to model red-light violation, yellow-light and green-light running in a two one-way intersection. Model is simulated using simHPN toolbox. The main advantage of a hybrid petri nets under simHPN is to utilize a different type of traffic characteristics such as macroscopic and microscopic behaviour and vehicle probability distribution random variable in case of red-light violation, yellow-light and green-light running. The random variables are obtained through a traffic count survey and analyze using KolmogorovSmirnov hypothesis test.
\end{abstract}

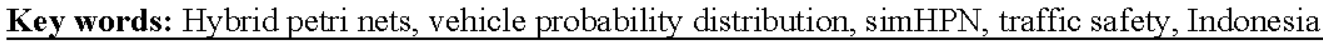

\section{INTRODUCTION}

Modeling and simulation of traffic control system in an intersection are the basic for the management of urban traffic area system. The traffic light arrangements are used to control the intersection, however the traffic flow can be influenced through driver information/guidance. In general, Petri Nets (PN) are tools for a research on control modeling for urban traffic network. Reseachers are focused on Vehicular Model, Roadlink Model and Intersection Model (Ramli and Harjono, 2011). Vehicular Interaction Modeling on the road has been investigated using colored PN (Hubner and Schnieder, 2010). Continuous PN (Tolba et al., 2005) are used to model a roadlink. Speed control mechanism in a highway roadlink has been studied using a batches PN (Demongodin, 2009).

Research on traffic signal control model at the intersection are investigated using PN (List and Cetin, 2004) on bus path routing using Colored Timed PN (Dotoli and Fanti, 2004) on macroscopic and microscopic behaviour using First Order Hybrid PN (Dotoli et al., 2008) on model predictive control using continuous PN (Julvez and Boel, 2010) on special vehicle priorities using Hybrid PN (Febbraro et al., 2004) on platoons of vehicles model using Hybrid PN (Vazquez et al., 2010). PN modelling for traffic safety at the intersection between roadway and railroad is examined using Stochastic PN (Ghazel, 2009).
Extended research on intersection model are towards modeling of red, yellow and green-light running of vehicles using deterministic stochastic timed PN. The probability of vehicles passing green, yellow and red signal of traffic lights is provided by a certain weight of the immediate transition (Farha and Schnieder, 2009). In other chases, the arrivals and departures probability of vehicles is assumed to follow a poisson (Comert and Cetin, 2011) and exponentially (Vazquez et al., 2010) distributed random variable.

The probability distribution of empirical data for red-light violation, yellow-light and green-light running can be discovered using Kolmogorov-Smirnov hypothesis testing (KS-test). KS-test are already used to examined the statistical characteristics of acoustic channel (Zhang et al., 2010). A detailed illustration of KS-test are shown in chemical experiments (Young, 1977). KS-test is a method to indicate the probability density function of data in comparison to statistical reference function such as: normal, uniform, exponential and poisson. The result can be used to make statistical hypothesis. If null hypothesis statement is retained than the empirical data pdf is the same as the statistical reference function (Montgomery, 2009). Confidence level of the KS-test result is characterized by the significance level $\alpha$. The default value for $\alpha$ is $5 \%$ and for the case of one-sided $\alpha$ is set to 0.025 .

A Red-Light Running (RLR) research has been conducted over a decade in terms of traffic safety. The

Corresponding Author: Mulyadi Sinung Harjono, Department of Electrical Engineering, Faculty of Engineering, Universitas Indonesia, 16424 Depok, Indonesia 
driver behaviours of RLR violators has been investigated (Porter and England, 2000). Daily fluctuation of RLR on hourly basis with linear regression analysis is examined under various condition such as Average Daily Traffic (ADT), number of approach lanes, speed limits, number of cross lanesand distance of preceding and following intersections (Hill and Lindly, 2003). RLR empirical study based on speed, distance parameters and headway is explored extensively with the aims to avoid the intersection collision (Zhang et al., 2008, 2012; Wang et al., 2009). In this case, the probability of RLR vehicles entries the dilemma zones with a certain speed is obtained and evaluated using Autoscope camera. A RFID based modelling and simulation for RLR using scilab has been investigated (Iswanjono et al., 2010). This study has two main goals:

- Proposed a new hybrid petri nets structure at the intersection to model red-light violation, yellow-light and green-light running in a two oneway intersection. Simulation of the model is running under simHPN toolbox (Julvez et al., 2012). The enhanced simHPN toolbox is capable to model a different types of vehicle probability distribution random variable that appear during arrivals and departure of vehicles at intersection

- Obtain random variables for the enhanced simHPN toolbox through traffic count survey for each cycle time of traffic lights during normal traffic condition. Traffic count data are analyzed using KolmogorovSmirnov hypothesis test

\section{Literature review}

Timed hybrid petri nets: Petri nets is a mathematische formalism and tools to describe the behaviour of discrete event system, see (Murata, 1989) for an introduction. State of the art of PN modelling in a real system leads to a discrete, hybrid and continuous structure (Silva et al., 2011).

In the case of traffic systems a timed Hybrid Petri Net class is used (Julvez et al., 2012). Its a system of a tuple, $\left\langle\mathrm{N}, \mathrm{m}_{0}\right.$, Type, $\left.\lambda\right\rangle$ where, $\mathrm{N}=\langle\mathrm{P}, \mathrm{T}$, Pre, Post $\rangle$ built the net structure with set of places $P$, set of transitions $T$, incidence matriks and initial marking. Type: $\mathrm{T} \rightarrow\{\mathrm{id}, \mathrm{pd}, \mathrm{dd}$, ic, pc\} formalize the time semantics of transitions and $\lambda: \mathrm{T} \rightarrow \mathrm{R}_{20}$, it shows a real number parameter coresponding to its semantics. The type abbreviation id is for discrete infinite server semantics; pd for discrete product server semantics, dd deterministic delay; ic for continuous infinite server semantics and pc for continuous product server semantics.
The marking evolution of PN structure is dependant on firing sequence of transitions. The enabling degree of a transitions is enab $\left(t_{j}, m\right)=\min _{p_{i} \in t_{j}}\left\lfloor m_{i} /\right.$ pre $\left.\left(p_{i}, t_{j}\right)\right\rfloor$. A transitions $t_{j}$ is enabled at $m$ iff enab $\left(t_{j}, m\right)>0$ and firing in any positive amount $\alpha$, where $\alpha \leq$ enab $\left(t_{j}, m\right)$. The firing leads to a new marking $\mathrm{m}^{\prime}=\mathrm{m}+\alpha \cdot \mathrm{C}\left(\mathrm{t}_{\mathrm{j}}\right)$ where, $\mathrm{C}=$ Post-Pre is a token flow matrix. Marking evolution depending on time for timed hybrid PN is determined by $m(\tau)=m_{0}+C \cdot \sigma(\tau)$ where, $\sigma(\tau)$ is the firing count vector at time $\tau$.

Time delays for discrete transition are depends on Type $T$. In case of id the time delay of transition $t_{j}(m)$ is an exponentially distributed random variable with paramater $\theta=\lambda_{i}$. enab $\left(t_{j}, m\right)$ where the integer enabling degree bescribe the number of active server of $t_{j}(\mathrm{~m})$. Enabled transition time delay for type dd is intended to fire after $1 / \lambda_{i}$ time units. The flow of a continuous transitions $\mathrm{t}_{\mathrm{j}}$ (Type: ic) is depends on $\mathrm{f}_{\mathrm{j}}=\lambda_{\mathrm{j}} \cdot$ enab $\left(\mathrm{t}_{\mathrm{j}}, \mathrm{m}\right)=$ $\lambda_{j} \cdot \min _{p_{i} \in t_{j}}\left\lfloor m_{i} /\right.$ pre $\left.\left(p_{i}, t_{j}\right)\right\rfloor$.

Conflict among discrete stochastic transitions (Type: id, pd) are solved by a race policy so that a transition with a smaller time delay will fire first. Conflicts among deterministic transitions (Type: $\mathrm{dd}$ ) are solved by a step of rules, i.e., first a race policy and if the conflict is persist then randomly choosing is carried out.

\section{MATERIALS AND METHODS}

Measurement and collection of data: Traffic counting aiming to measure the probability distribution of traffic flow in intersection were obtain at two intersections, located at the crossing between Jalan Kyai Haji Wahid Hasyim-Jalan Mohammad Husni Thamrin and Jalan Kebon Sirih-Jalan Mohammad Husni Thamrin, Jakarta Indonesia. The signalized intersections consists of 12-lanes and 4-lanes crossing road and equiped with an actuated traffic control system using historical data. The traffic lights are not equipped with automatic countdown timer. A right flow of vehicles are not permitted. Only the yellow light signal aspect is constant $2 \mathrm{sec}$.

The Traffic Counting (TC) is done for each cycle of traffic light signal aspect. The TC's are started from the stop line of the traffic lights and do not distinguish between the classification of vehicles. The data is observed in a normal traffic condition, therefore weekends, monday and busy hours are avoided.

Figure 1 shows the observed data for 12 lanes red-light violation as 12LaneRLR; 4-lanes red-light violation as 4LaneRLR; 12-lanes yellow-light running as 12LaneYLR and 4-lanes yellow-light running as 4Lane YLR. The highest outlier data in 12LaneRLR and 4LaneRLR are the number of no red-light violations (incidence 0). About 12LaneYLR and 4Lane YLR indicate 


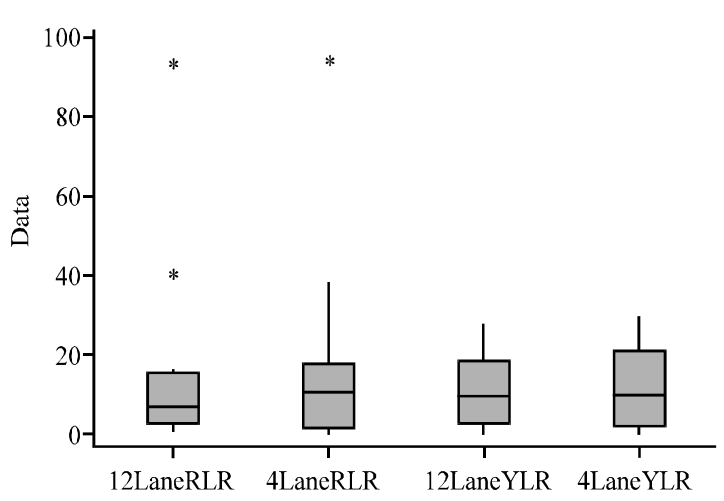

Fig. 1: Data observation result

that there is none of the vehicle stops during a yellowafter a green light signal aspect and there is no vehicle flow during the yellow-after a red light signal aspect. Whiskers range in 4lanesRLR is breiter than in 12 lane showing that the trends of RLR is greater in smaller lane croosing road.

Distribution analysis test: Analysis of the empirical data is carried out using Kolmogorov-Smirnov hypothesis test. The KS-test function is capable to test the empirical data distributions and the parameters of the hypothesized distributions (normal, exponential and poisson probability density function pdf). The significance level $\alpha$ (p-value) for a one tailed test is 0.025 . The null hypothesis is rejected if $\mathrm{p}<0.025$. The Kolmogorov-Smirnov Z (K-S Z) is the largest difference (absolute value) between the observed and theoretical cumulative distribution functions.

In the case of red-light violation, the null probability distribution hypothesis are retained only for the normal and exponential pdf. The hypothesis of poisson pdf for a red-light violation distribution is rejected $(\mathrm{p}<0.025)$. KS-Z result shows that exponential pdf is more appropriate distribution for a red-light violation than the normal pdf.

KS-test for the yellow-light running indicate that the empirical data are all retained the null hypothesis (normal, exponential and poisson pdf's). In this case, the poisson pdf can be used as a new candidate for the distribution of yellow-light running. Although, based on the KS-Z value it shown that a exponential pdf is more suitable. Generally, the KS-test result has shown that the distributions are not depends on the lane size to be cross and for all cases the empirical data have a normal distribution.

The p-value for Yellow Light Running (YLR) showing that a poisson distribution is retain null hypothesis as well as a normal and a exponential distribution. KS-Z analysis for YLR showing that a poisson distribution have a greater value difference between standard poisson and

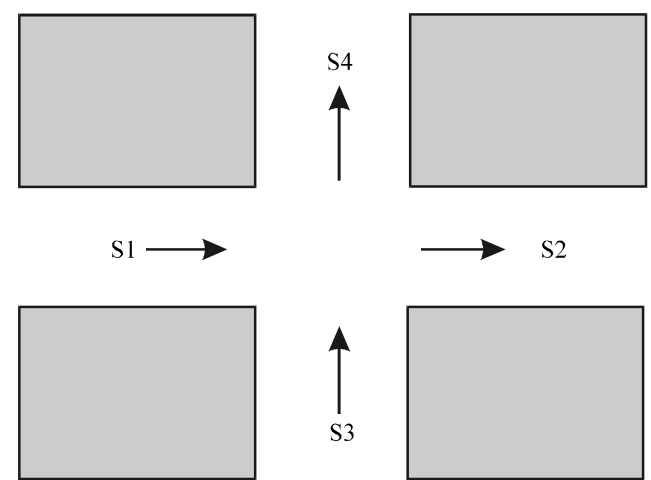

Fig. 2: A model of two one-way intersection

empirical value in contrast to normal and exponential distribution that have almost the similar value. However, analysis of critical point at zero for YLR showing that none of the vehicles is stopping during the yellow-light. The choosen pdf shall then start with incidence zero as zero as shown by a poisson distribution so that an exponential pdf is not preferable for this case. The greenlight running is assummed has the same pdf as YLR due to the same behaviour that the vehicles is not stopping during green-light aspect and the red-light violation has a exponential pdf.

\section{Intersection modelling}

Modeling of traffic at intersection using hybrid petri nets: Based on earlier empirical data analysis we proposed a new structural petri nets model and simulation for a a signalized intersection of two one-way street in Fig. 2 capable for a red-light violation model based on exponential pdf, furthermore a yellow-light and a green-light running model based on poisson pdf using Hybrid Petri Nets (HPN). Basic definition for HPN used for the model are taken from the simHPN toolbox (Julvez et al., 2012) running under MATLAB.

The incoming flows of vehicle into the intersection are modelled using continuous petri nets transitions. The model has a macroscopic behaviour that deal for each road segment with the parameters: average flow rate and vehicle density. Therefore, it is assumed in continuous model that the average flow rate is constant and the vehicle is homogeneously distributed in an intersection road segment. Upon entering the intersection, dynamic modeling of vehicles crossing the intersection require a microscopic model using discrete petri nets transitions that have a type of infinite server semantics capable for time delay based on exponential pdf or poisson pdf. A microscopic model deal with timed availability of road segments and vehicles. The intersection is controlled by a traffic lights with a specific cycle time which is discrete 
and deterministically delay transition petri nets. The structure of petri nets for the desired intersection model alltogether built then a hybrid petri nets.

Figure 3 shows the constant incoming flow rate $\lambda_{i}$ through a continuous transition $t_{c i}$ queueing in place $\mathrm{p}_{\mathrm{i}}$. Upon entering the intersection a discrete transition $t_{d i}$ is enabled through place $p_{i}$. The enabled infinite server semantics transition $t_{\mathrm{di}}$ will fire following an exponentially or poisson distributed random time delay with parameter $\lambda_{i} \cdot$ enab $\left(t_{d i}, m\right)$. The integer enabling enab $\left(t_{d i}, m\right)$ of transition $t_{d i}$ showing the number of active server of $t_{d i}$ at marking $m$ that departing place $p_{i}$ and arriving in place $p_{i+1}$ with a constant rate $\lambda_{1}$. The number of vehicles moving into the intersection road segment is the arrived number of active server that appear in place $p_{i+1}$. So that the number of vehicle in a red-light violation can be generated using exponential pdf, furthermore a yellow-light running and a green light flow using poisson pdf, respectively. Conflict between infinite server semantics discrete transitions are solved using racing policy, i.e., the firing priority of discrete transition in conflict is based on smaller time delay among them.

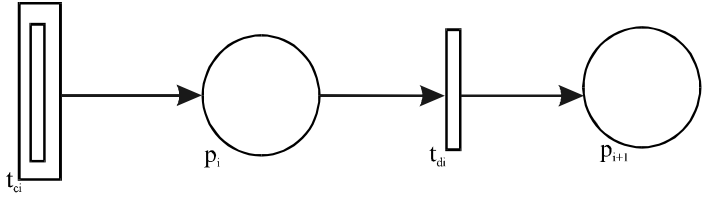

Fig. 3: Continuous to discrete transition
A new structure HPN Model for the signalized intersection with a red-light violation, yellow-light and green-light running model is shown in Fig. 4. The incomming flow into the intersection is modelled by a continuous petri nets $\left\{\mathrm{p}_{1}{ }^{1}, \mathrm{p}_{2}{ }^{1}\right\}$ and $\left\{\mathrm{t}_{1}{ }^{1}\right\}$. The flow of vehicles before crossing the intersection is modelled through the continuous transitions $\left\{\mathrm{t}_{2}{ }^{1}\right.$ or $\mathrm{t}_{3}{ }^{1}$ or $\left.\mathrm{t}_{4}{ }^{1}\right\}$. The $\left\{\mathrm{t}_{2}{ }^{1}, \mathrm{t}_{3}{ }^{1}, \mathrm{t}_{4}{ }^{1}\right\}$ are dedicated for vehicles running greenlight, yellow-light or red-light violation, respectively. Each of these continuous transition is enabled, depends on the corresponding traffic light signal aspect in place $\left\{\mathrm{p}_{1}, \mathrm{p}_{2}\right.$, $\left.\mathrm{p}_{3}\right\}$. Places $\left\{\mathrm{p}_{3}{ }^{1}, \mathrm{p}_{4}{ }^{1}, \mathrm{p}_{5}{ }^{1}\right\}$ are the queueing vehicles before entering the intersection and leaving transitions $\left\{\mathrm{t}_{2}{ }^{1}, \mathrm{t}_{3}{ }^{1}\right.$, $\left.\mathrm{t}_{4}{ }^{1}\right\}$, respectively. The vehicle generation upon entering the intersection are follows through a discrete transition with the type of infinite server semantics $\left\{\mathrm{t}_{5}{ }^{1}\right.$ or $\mathrm{t}_{6}{ }^{1}$ or $\left.\mathrm{t}_{7}{ }^{1}\right\}$ that has been enabled by $\left\{\mathrm{p}_{\mathrm{r}}\right\}$ and $\left\{\mathrm{p}_{3}{ }_{3}, \mathrm{p}_{4}{ }^{1}\right.$ or $\left.\mathrm{p}_{5}{ }^{1}\right\} .\left\{\mathrm{p}_{\mathrm{r}}\right\}$ represent the availability of the shared road segment at the intersection and has a constant maximal number of marking $m_{r}$. The value of marking $m_{r}$ is depends on the size of intersection in this case is set to $8 .\left\{p_{r}\right\}$ connected to all transition transition if the segment is available. If a vehicle crossing the intersection, then number of $\mathrm{m}_{\mathrm{r}}$ reduced. After vehicles leaving the intersection, the number of $\mathrm{m} \mathrm{m}_{\mathrm{r}}$ increase back through transitions $\left\{\mathrm{t}_{8}{ }^{1}\right.$ or $\left.\mathrm{t}_{8}{ }^{2}\right\}$. The firing sequence coming through $\left\{\mathrm{t}_{5}{ }^{1}\right\}$ or $\left\{\mathrm{t}_{6}{ }^{1}\right.$ or $\left.\mathrm{t}_{7}{ }^{1}\right\}$ has an exponential or poisson probability distribution time delay function characteristics, respectively.

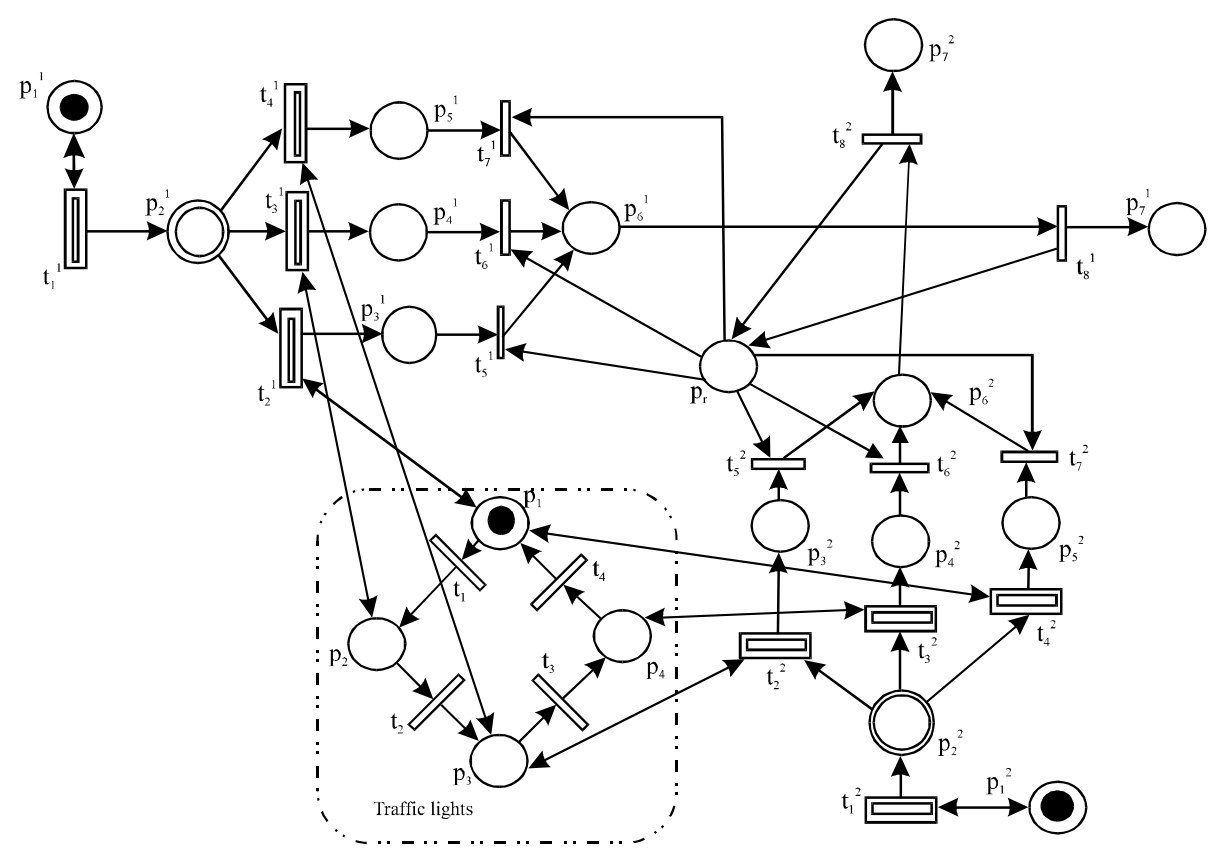

Fig. 4: HPN Model for signalized intersection 
The generated vehicles coming into the intersection are placed $\left\{\mathrm{p}_{6}{ }^{1}\right\}$ as a discrete petri nets. The accumulation of vehicles leaving the intersection, can be seen in $\left\{\mathrm{p}_{7}{ }_{7}^{1}\right\}$.

The structure $\left\{\mathrm{p}_{1}, \mathrm{p}_{2}, \mathrm{p}_{3}, \mathrm{p}_{4}\right\}$ and $\left\{\mathrm{t}_{1}, \mathrm{t}_{2}, \mathrm{t}_{3}, \mathrm{t}_{4}\right\}$ built a Traffic Lights (TL) Model. The places are enabling the flow of traffic. A marking in $\left\{\mathrm{p}_{1}\right\}$ enabled the green light for queue $\left\{\mathrm{p}_{2}{ }^{1}\right\}$ and vehicle flow through $\left\{\mathrm{t}_{2}{ }_{2}\right\}$, however its enabled the red light for queue $\left\{\mathrm{p}_{2}{ }^{2}\right\}$ and violation flow through $\left\{\mathrm{t}_{4}^{2}\right\}$. The type of TL Transition Model is deterministic delay and describe the traffic phase. Places $\left\{\mathrm{p}_{2}, \mathrm{p}_{4}\right\}$ illustrated the yellow light.

The HPN structure $\mathrm{p}_{\mathrm{x}}{ }^{2}$ and $\mathrm{t}_{\mathrm{s}}{ }^{2}$ showing the model of traffic for the second one-way street from road segment S3 to S4 in the same way as the structure node with a subscript 1. Conflicts occurs between vehicles from green-light running $\left\{\mathrm{t}_{2}{ }^{\mathrm{B}}\right\}$ and red-light violation $\left\{\mathrm{t}_{4}{ }^{\mathrm{y}}\right\}$ are solved by a racing policy. The discrete transition with a smaller time delay will fire first.

Time delay time setting for $\left\{\mathrm{t}_{1}{ }_{1}, \mathrm{t}_{2}{ }^{1}, \mathrm{t}_{3}{ }^{1}, \mathrm{t}_{4}{ }^{1}\right\}$ are $(0.5 ; 1$; $0.6 ; 0.2) \mathrm{sec}$, for $\left\{\mathrm{t}_{1}{ }^{2}, \mathrm{t}_{2}{ }^{2}, \mathrm{t}_{3}{ }^{2}, \mathrm{t}_{4}{ }^{2}\right\}$ are $(0.5 ; 1 ; 0.4 ; 0.1) \mathrm{s}$, for $\left\{\mathrm{t}_{5}{ }^{1}, \mathrm{t}_{6}{ }^{1}, \mathrm{t}_{7}{ }^{1}, \mathrm{t}_{8}{ }^{1}, \mathrm{t}_{5}{ }^{2}, \mathrm{t}_{6}{ }^{2}, \mathrm{t}_{7}{ }^{2}, \mathrm{t}_{8}{ }^{2}\right\}$ are $(1 ; 1 ; 1 ; 1,1 ; 1 ; 1 ; 1) \mathrm{sec}$ and the deterministic delay for the TL $\left\{\mathrm{t}_{1}, \mathrm{t}_{2}, \mathrm{t}_{3}, \mathrm{t}_{4}\right\}$ are $(20,5,20,5) \mathrm{sec}$.

\section{RESULTS AND DISCUSSION}

Enhancement of the procedure Update clocks in Algorithm 1 (Julvez et al., 2012) shown in Fig. 5 is necessary to acommodate a discrete transition under infinite server semantics with a time delay based on poisson besides exponential probabilistic density characteristics. This leads to a new type of infinite server semantics, i.e., id (poisson) besides the previous id (exponential). The variable clocks bescribe the internal firing time schedule for each the discrete transition. Variable $\mathrm{S}$ represent a set of all enabled transition.

Figure 6 shows the marking evolution of $\mathrm{m} 2(\mathrm{~m} 9)$ corresponds to macroscopic, queue dynamics of vehicles in $\mathrm{p}_{2}{ }^{1}\left(\mathrm{p}_{2}{ }^{2}\right)$, respectively. Besides, the marking m6 ilustrate the discrete dynamic of vehicles entering the intersections in the direction S1-S2. In this simulation $\mathrm{m} 16$ showing the green phase of the traffic light for $\mathrm{m} 2$.

Figure 7 simulate the generated vehicle through green-light, yellow-light running and red-light violation represent by $\mathrm{m} 6$. The marking $\mathrm{m} 7$ showing the accumulation of vehicle leaving the intersection in the road segment S2. Marking $\mathrm{m} 15$ is the marking evolution of $\mathrm{p}_{\mathrm{r}}$ and $\mathrm{m} 16$ showing the green phase of the traffic light for $\mathrm{m} 2$.

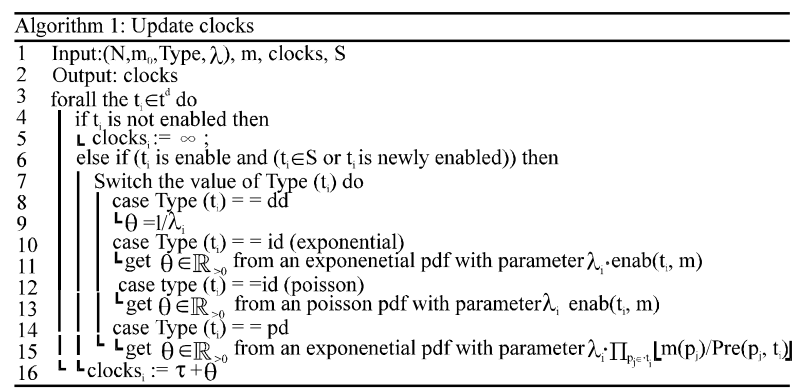

Fig. 5: Enhancement of Algorithm 1 (Julvez et al., 2012)

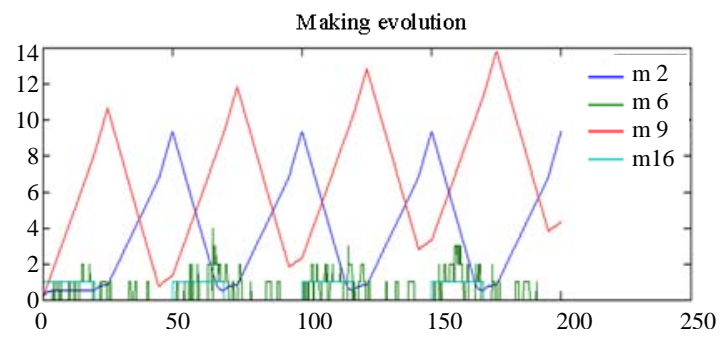

Fig. 6: Queue dynamics

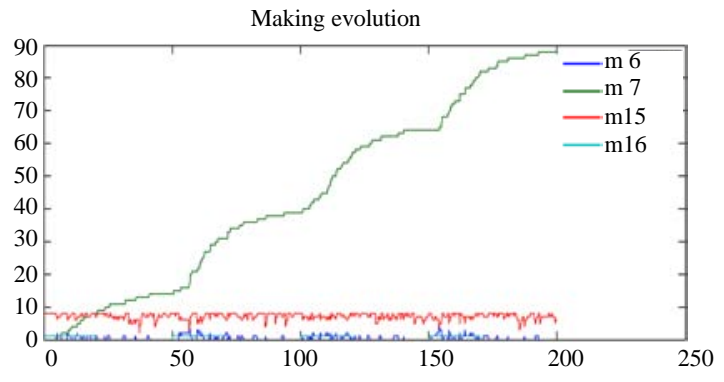

Fig. 7: Resource sharing in $\mathrm{m} 15$

\section{CONCLUSION}

Experiments and analysis using Kolmogorov-Smirnov hypothesis test shows that Red-Light violation (RLR) has the normal and exponential pdf. A poisson pdf hypothesis for RLR is rejected with the logical explanation that the zero incidence is occurred in RLR. It means that the people tends not to violate a red light aspect. On the other hand, Yellow-Light Running (YLR) analyses indicate that a normal, exponential and poisson pdf is eligible. However in the traffic count survey indicate that there is no zero incidence so that poisson pdf is more appropriate. For the same reason the green-light running has a poisson pdf as a YLR.

A new structure of hybrid petri net model for signalized intersection with capability to represent a red-light violation, yellow-light and green light running is developed running under simHPN. An enhancement of 
procedure for update clocks in Algorithm 1 of simHPN has been made, to accommodate the time delay transition with a poisson probability density characteristics for type of infinite server semantics.

\section{REFERENCES}

Comert, G. and M. Cetin, 2011. Analytical evaluation of the error in queue length estimation at traffic signals from probe vehicle data. Proceedings of the IEEE Transactions on Intelligent Transportation Systems, Vol. 12, June 2011, Columbia, SC, USA, pp: 563-573.

Demongodin, I., 2009. Modeling and analysis of transportation networks using Batches Petri nets with controllable batch speed. Proceedings of the 30th International Conference on Applications and Theory of Petri Nets, Vol. 30, June 22-26, 2009, Paris France, pp: 204-222.

Dotoli, M. and M.P. Fanti, 2004. An urban traffic network model via coloured timed petri nets. Control Eng. Pract., 14: 1213-1229.

Dotoli, M., M.P. Fanti, A. Giua and C. Seatzu, 2008. First-order hybrid petri nets. An application to distributed manufacturing systems. Nonlinear Anal: Hybrid Syst., 2: 408-430.

Farha, A. and E. Schnieder, 2009. Modelling of traffic safety and operation in urban networks using DSTPN. Proceedings of the 12th IFAC Symposium on Transportation Systems, Septmber 2-4, 2009, Redondo Beach, CA, USA, pp: 513-518.

Febbraro, A.D., D. Giglio and N. Sacco, 2004. Urban traffic control structure based on hybrid petri nets. Proceeding of the IEEE Transactions on Intelligent Transportation Systems, Vol. 5, December 6, 2004, Italy, pp: 224-237.

Ghazel, M., 2009. Using stochastic petri nets for level-crossing collision risk assessment. Proceedings of the IEEE Transactions on Intelligent Transportation Systems, Vol. 10, December 2009, France, pp: 668-677.

Hill, S.E. and J.K. Lindly, 2003. Red light running prediction and analysis. University Transportation Center for Alabama, UTCA Report Number 02112.

Hubner, M. and E. Schnieder, 2010. Engineering of Car2Car interactions by means of colored petri net road model. Proceedings of the 12th World Conference on Transportation Research, July 11-15, 2010, Lisbon, Portugal, pp: 1-17.
Iswanjono, B. Budiarjo and K. Ramli, 2010. Simulation for RFID-based red light violation detection: Violation detection and flow prediction. Proceedings of the 2nd International Conference on Computer Research and Development, May 7-10, 2010, Kuala LumpurMalaysia, pp: 742-747.

Julvez, J. and R.K. Boel, 2010. A continuous petri net approach for model predictive control of traffic systems. Proceedings of the IEEE Transactions on Systems, Man and Cybernetics, Part A: Systems and Humans, Vol. 40, July 2010, Zaragoza, pp: 686-697.

Julvez, J., C. Mahulea and C. Vazquez, 2012. SimHPN: A MATLAB toolbox for simulation, analysis and design with hybrid petri nets. Nonlinear Anal: Hybrid Syst., 6: 806-817.

List, G.F. and M. Cetin, 2004. Modeling traffic signal control using petri nets. IEEE Trans. Intell. Transp. Syst., 5: 177-187.

Montgomery, D.C., 2009. Design and Analysis of Experiment. 7th Edn., John Wiley and Sons, Inc., New York, pp: 34.

Murata, T., 1989. Petri nets: Properties, analysis and applications. Proc. IEEE, 77: 541-580.

Porter, B.E. and K.J. England, 2000. Predicting red-light running behavior: A traffic safety study in three urban settings. J. Safety Res., 31: 1-8.

Ramli, K. and M.S. Harjono, 2011. Deadlock-freeness properties of petri nets in urban traffic network: A survey. Proceedings of the International Symposium on Chaos Revolution in Science, Technology and Society, February 21-22, 2011, Depok, Indonesia.

Silva, M., J. Julvez, C. Mahulea and C.R. Vazquez, 2011. On fluidization of discrete event models: Observation and control of continuous petri nets. Discrete Event Dyn. Syst., 21: 427-497.

Tolba, C., D. Lefebvre, P. Thomas and A. El Moudni, 2005. Continuous and timed petri nets for the macroscopic and microscopic traffic flow modelling. Simulat. Modelling Practice Theory, 13: $407-436$.

Vazquez, C.R., H.Y. Sutarto, R.K. Boel and M. Silva, 2010. Hybrid petri net model of a traffic intersection in a urban network. Proceedings of the IEEE Internatioal Conference Control Applications, September 8-10, 2010, Yokohama, pp: 658-664.

Wang, L., L. Zhang, W. Zhang and K. Zhou, 2009. Red light running prediction for dynamic all-red extension at signalized intersection. Proceedings of the 12th Instructional Technology Strategies Conference, October 4-7, 2009, St. Louis, USA., pp: 1-5. 
Young, I.T., 1977. Proof without prejudice: Use of the kolmogorov-smirnov test for the analysis of histograms from flow systems and other sources. J. Histochem. Cytochem., 25: 935-941.

Zhang, J., J. Cross, and Y.R. Zheng, 2010. Statistical channel modeling of wireless shallow water acoustic communications from experiment data. Proceedings of the Military Communications Conference, October 31, 2010, San Jose, CA., USA., pp: 2412-2416.
Zhang, L., K. Zhou, W. Zhang and J.A. Misener, 2008. Empirical observations of red light running at arterial signalized intersection. Path Tech. Note, http://trid.trb.org/view.aspx?id=887439.

Zhang, L., L. Wang, K. Zhou and W. Zhang, 2012. Dynamic all-red extension at a signalized intersection: A framework of probabilistic modeling and performance evaluation. IEEE Trans. Intell. Transp. Syst., 13: 166-179. 